\title{
Biomarcadores de cáncer oral en saliva
}

\section{Salivary analysis of oral cancer biomarkers}

\author{
Madera Anaya $\mathrm{MV}^{*}$
}

\section{RESUMEN}

Esta revisión muestra los principales biomarcadores de cáncer oral en saliva. El aspecto clínico y el grado de displasia de las lesiones precancerosas de la cavidad bucal sugieren su capacidad de malignidad; sin embargo, éstas generalmente son diagnosticadas en estadios avanzados, disminuyendo la probabilidad de supervivencia, lo que justifica el diseño de nuevas pruebas diagnósticas que determinen el grado de alteración celular, permitan comprender el proceso degenerativo en el cáncer y establezcan diagnósticos precoz. Esta búsqueda para mejorar los métodos diagnósticos, apunta a que sean sensibles, específicos y menos invasivos, por lo cual el estudio de diferentes biomarcadores en saliva que desde una perspectiva molecular proporcionan información adicional al examen clínico e histopatológico, es considerada como una alternativa eficaz y más cómoda con respecto a los ensayos en sangre. Los biomarcadores que se han descrito en saliva algunos mostrando mayor relación con la carcinogénesis oral son: Ciclina D1, cyfra 21-1, endotelina-1, galectinas 1, 3 y 7, Ki67, lactato deshidrogenasa, metaloproteinasas 2 y 9, proteína p53, proteína de unión a calcio (S100P) y telomerasa.

Palabras clave: Cáncer oral, biomarcadores, saliva.

\section{SUMMARY}

This review shows the main oral cancer biomarkers in saliva. The clinical appearance and the degree of dysplasia, precancerous lesions of the oral cavity suggests its ability to malignancy, but these are usually diagnosed in advanced stages, decreasing the likelihood of survival, justifying the design of new diagnostic tests to determine the degree of cell alteration as to understand the degenerative process in cancer diagnosis and establish early. This search for improved diagnostic methods, aims to be sensitive, specific and less invasive, so the study of biomarkers in saliva from a molecular perspective provide additional information to clinical and histopathological examination is considered as a more comfortable and effective to establish a diagnosis. Biomarkers that have been described in saliva some showing more related to oral carcinogenesis are cyclin D1, Cyfra 21-1, Endothelin-1, Galectins 1, 3 and 7, Ki67, Lactate dehydrogenase, Metalloproteinases 2 and 9, p53 protein, protein calcium-binding (S100P) and Telomerase.

Key words: Oral cancer, biomarkers, saliva.

Fecha de recepción: 25 de febrero de 2013.

Aceptado para publicación: 22 de abril de 2013.

* Odontólogo. Magíster en Bioquímica (cand.). Universidad de Cartagena, Colombia. Magíster en Epidemiología Clínica (cand.). Universidad de la Frontera. Temuco, Chile.

Madera Anaya MV. Biomarcadores de cáncer oral en saliva. Av. Odontoestomatol 2013; 29 (6): 293-302.

\section{INTRODUCCIÓN}

El cáncer oral es una enfermedad en donde las células de la cavidad bucal se dividen sin control y pueden invadir otros tejidos; este se desarrolla por múltiples factores: ambientales, inmunológicos, nutricionales, hábitos y estilos de vida (1). A nivel celular surge como consecuencia de trastornos genómicos generados por aberraciones cromosómicas, activación de oncogenes e inactivación de genes su- 
presores tumorales, originando células con características proliferativas e invasivas $(2,3)$.

En los últimos años la prevalencia de cáncer oral ha aumentado, se estima que en total 350.000 nuevos casos se registran anualmente en todo el mundo, de estos el 10\% ocurren en los Estados Unidos (4); el riesgo es más alto en hombres, en mayores de 40 años, fumadores, consumidores de alcohol y en personas con antecedentes de cáncer de cabeza y cuello $(5,6)$.

El cáncer oral más frecuente es el carcinoma de células escamosas presentándose comúnmente en los labios, lengua, orofaringe, paladar, mucosas yúgales y piso de boca (7). El diagnóstico de estas lesiones empieza con el examen clínico y es confirmado con el estudio histopatológico que muestra la presencia y grado de displasia; sin embargo, no permiten determinar el grado de invasión y el potencial metastásico (8).

Generalmente, este tipo de cáncer puede iniciar con una lesión de mancha blanca o una úlcera, que no tiene un factor etiológico claro asociado ni resolución satisfactoria a corto plazo, hay aumento del volumen de la lesión, cronicidad, cambios morfológicos y linfadenopatías (9). Histológicamente la severidad se establece basada en el grado de diferenciación, en donde se tiene en cuenta el nivel de queratinización, pleomorfismo celular y nuclear y la actividad mitótica (10).

Se ha establecido que la vida media de supervivencia de estos pacientes es de 5 años tras el diagnóstico que generalmente se realiza sobre lesiones de fase avanzada (2). Esto hace necesario buscar pruebas que establezcan de manera precoz el riesgo de padecer esta enfermedad o identifiquen lesiones precancerosas; en este sentido, se deben emplear de forma selectiva, otros exámenes más específicos, que permitan valorar las alteraciones celulares y establecer un diagnóstico precoz de esta patología. Para la realización de estos exámenes existen diferentes biomarcadores celulares y tisulares que desde una perspectiva molecular, proporcionan información adicional a la obtenida en el examen clínico y el estudio histopatológico (11-14).

Aunque existe un creciente esfuerzo dedicado a la investigación del cáncer oral, centrándose en la identificación de indicadores biológicos para el diagnósti- co y la agresividad biológica, son pocos los estudios que han examinado biomarcadores tumorales en la saliva. En el presente trabajo de revisión se muestran los principales marcadores de cáncer oral en saliva.

\section{SALIVA}

La saliva es un fluido corporal de la cavidad oral formado por una mezcla compleja de proteínas y otras moléculas que se originan a partir de varias fuentes (15). Las propiedades bioquímicas y fisicoquímicas de la saliva contribuyen a sus numerosas funciones entre las cuales están lubricación, capacidad tampón, protección antimicrobiana, digestión de alimentos, mantenimiento de la integridad de la mucosa, sostenimiento de la salud oral y general $(16,17)$.

Ésta se ha utilizado como un medio de investigación durante décadas en los pacientes con enfermedades locales y sistémicas, debido a que está en contacto directamente con la mucosa oral, la descamación de las células y sus productos pueden ser detectados, lo que hace posible estudiar marcadores tumorales en saliva de pacientes con cáncer oral (18-23). Entre las ventajas en comparación con las muestras de sangre se han propuesto que el método de recolección es seguro, indoloro y no traumático, la técnica de recolección es fácil de manipular, el equipo de recolección es simple y la muestra de saliva puede ser tomada varias veces sin causarle incomodidad al paciente $(24,25)$. Hu (26) sostiene que la utilización de la saliva en comparación con la sangre puede demostrar marcadores más sensible y específicos para las enfermedades orales tales como carcinoma oral de células escamosas.

La utilización de la saliva se considera una alternativa no invasiva con relación a las muestras de sangre o biopsias, convirtiéndose en una efectiva modalidad para el diagnóstico y predicción del pronóstico de cáncer oral a través de un monitoreo a pacientes en tratamiento $(27,28)$.

\section{BIOMARCADORES EN SALIVA}

Un biomarcador es una característica que es medida y evaluada objetivamente como un indicador de pro- 
cesos biológicos normales, patológicos o respuestas farmacéuticas a una intervención terapéutica (29). En su forma más precisa, el término debe hacer referencia a una sola especie molecular que está presente en las muestras de un sujeto con una determinada enfermedad o condición (25).

Basados en el proceso carcinogénico según Chimenos (8) estos se pueden agrupar por familia en biomarcadores decrecimiento tumoral, supresión tumoral, angiogénesis, Invasión tumoral, celulares de superficie, intracelulares, enzimáticos y derivados del ácido araquidónico.

Entre los biomarcadores de cáncer que se han descrito en saliva están: 8-oxoguanina ADN glicosilada, ácido hidroxieicosatetraenoico, carbonilos, ciclina D1, cistatina truncada SA-I, Cyfra 21-1. La proteína fosfatasa dual específica 1 (DUSP1), endotelina-1, fosfato sérico, galectinas 1,3 y 7 , inhibidor de la proteasa serina mamaria (Maspin), interleuquina 1 beta, interleuquina 8, Ki67, lactato deshidrogenasa, proteína M2BP, metaloproteinasas 2 y 9, proteína p53, proteína de unión a calcio (S100P), proteína autotrasportadora 1 (SAT1), telomerasa y transferrinas $(4,20,28,30-42)$ (tabla 1).

Teniendo en cuenta que el diagnóstico de cáncer oral se realiza en estadios avanzados, se hace necesario el estudio de biomarcadores que se expresen en estadios iniciales de la enfermedad para que puedan ser útiles en el diagnóstico precoz, en este sentido conociendo que el proceso cancerígeno se inicia con cambios a nivel celular es pertinente evaluar aquellos que se han reportado en saliva y están involucrados en el ciclo celular, apoptosis o procesos relacionados; entre los más reportados se encuentran los siguientes:

\section{MARCADORES DE CRECIMIENTO TUMORAL}

\section{Telomerasa}

En varios cánceres, la habilidad de dividirse sin límite es logrado por la producción de una enzima llamada telomerasa, la cual ayuda a los cromosomas para que no se acorten. Ésta es una proteína normal que está presente en las células durante el desarrollo del feto. En la mayoría de células de un adulto, la telomerasa no está presente ya que la enzima no está siendo expresada. Sin embargo, en algunas células cancerosas esta función es necesaria reactivando el gen que codifica la telomerasa (43).

Las investigaciones sobre el carcinoma escamoso celular de cabeza y cuello mostraron que la actividad de la telomerasa se expresó en el $75 \%$ de los tejidos tumorales relacionada con el pronóstico, así que la detección de la actividad de la telomerasa podría ser de potencial valor diagnóstico del cáncer oral (44); sin embargo, para autores como Zhong (40) aunque la expresión positiva de la actividad de la telomerasa en la saliva fue del $75 \%$, no existe diferencia entre los pacientes en estadios iniciales y finales de la lesión, así como entre los pacientes con y sin metástasis a los ganglios linfáticos, por lo tanto, el valor diagnóstico es limitado, considerándose solo como un marcador coadyuvante para el diagnóstico de cáncer oral.

\section{Endotelina-1 (ET-1)}

La ET-1 pertenece a la familia de endotelinas, potentes vasoconstrictores que participan no sólo en la biología vascular, sino también en diversos procesos incluyendo la inflamación, la cicatrización de heridas y la carcinogénesis. Los niveles elevados de ET-1 mRNA y proteína de ET-1 han sido reportados en el cáncer oral (45).

La expresión de endotelinas y sus receptores se asocia con un alto grado de agresividad, invasión y metástasis. Hay aumento de los niveles sistémicos de las endotelinas en los pacientes con metástasis en los ganglios linfáticos. Los mecanismos por los cuales las endotelinas inducen un fenotipo invasivo incluyen la interacción entre las células tumorales, los macrófagos infiltrantes y el microambiente del tumor. Esta compleja interacción conduce a la modulación de las metaloproteinasas de matriz, la expresión de citoquinas, se infiltran en la activación inmune, la apoptosis y la expresión de las mismas endotelinas (46).

Autores han reportado una elevación significativa de ET-1 en los carcinomas orales (35); sin embargo, 
TABLA 1.- BIOMARCADORES SALIVALES Y TÉCNICAS EMPLEADAS PARA SU ANÁLISIS

\begin{tabular}{|c|c|c|c|}
\hline Biomarcador & Técnica & Observaciones & Autor y año \\
\hline $\begin{array}{l}\text { 8-Oxoguanina ADN } \\
\text { glicosilada }\end{array}$ & ELISA & Disminuye. & Shpitzer, 2009 \\
\hline $\begin{array}{l}\text { Ácido } \\
\text { hidroxieicosatetraenoico }\end{array}$ & $\begin{array}{l}\text { Cromatografía de gases, } \\
\text { espectrometría de masas } \\
\text { y la dilución de isótopos } \\
\text { estables. }\end{array}$ & $\begin{array}{l}\text { Aumentan, están involucrados en los } \\
\text { procesos de metástasis e invasión tumoral. }\end{array}$ & Metzger, 1995 \\
\hline Carbonilos & Oxidación de proteínas & $\begin{array}{l}\text { Aumentados, indica daño oxidativo de } \\
\text { proteínas. }\end{array}$ & Shpitzer, 2009 \\
\hline Ciclina D1 & ELISA & $\begin{array}{l}\text { Aumenta, se relaciona con la progresión } \\
\text { celular y mal pronostico. }\end{array}$ & Shpitzer, 2009 \\
\hline Cistatinatruncada SA-I & SELDITOF & Aumenta, se relaciona con estadios de tumor. & Shintani, 2010 \\
\hline Cyfra 21-1 & ELISA & Se relacionada con la recurrencia. & Zhong, 2007 \\
\hline DUSP1 & PCR & Se aumentan en el cáncer oral. & Brinkmann, 2012 \\
\hline Endotelina-1 & ELISA & Buen marcador para diagnosticar liquen plano. & Cheng, 2011 \\
\hline Fosfato sérico & ELISA & Disminuye. & Shpitzer, 2009 \\
\hline Galectinas 1,3 y 7 & $\begin{array}{l}\text { Análisis } \\
\text { inmunohistoquímico }\end{array}$ & $\begin{array}{l}\text { Aumentada, relacionadas con la progresión } \\
\text { del tumor. }\end{array}$ & Alves, 2011 \\
\hline Maspin & ELISA & $\begin{array}{l}\text { Su disminución se relaciona con el } \\
\text { crecimiento, progresión y metástasis. }\end{array}$ & Shpitzer, 2009 \\
\hline Interleuquina 1 beta & ELISA- PCR & Se aumentan en el cáncer oral. & $\begin{array}{l}\text { Brinkmann, } 2012 \\
\text { Elashoff, } 2012\end{array}$ \\
\hline Interleuquina 8 & ELISA- PCR & Se aumentan en el cáncer oral. & $\begin{array}{l}\text { Brinkmann, } 2012 \\
\text { Elashoff, } 2012\end{array}$ \\
\hline Ki67 & ELISA & $\begin{array}{l}\text { Aumentan, relacionadas con la progresión } \\
\text { celular y mal pronostico }\end{array}$ & Shpitzer, 2009 \\
\hline Lactato deshidrogenasa & Espectrofotometría & $\begin{array}{l}\text { Indicador de necrosis celular, se relaciona } \\
\text { con el mal pronóstico y la recurrencia. }\end{array}$ & Shpitzer, 2009 \\
\hline M2BP & ELISA & $\begin{array}{l}\text { Se eleva principalmente en estadios } \\
\text { iniciales del cáncer oral. }\end{array}$ & $\begin{array}{l}\text { Brinkmann, } 2012 \\
\text { Elashoff, } 2012\end{array}$ \\
\hline Metaloproteinasas 2 y 9 & ELISA & Aumentan, relacionada con la metástasis. & $\begin{array}{l}\text { Shpitzer, 2007, } \\
2009\end{array}$ \\
\hline p53 & PCR & $\begin{array}{l}\text { Su disminución se asocia a la capacidad } \\
\text { de aberraciones celulares. }\end{array}$ & Liao, 2000 \\
\hline S100P & $\begin{array}{l}\text { Western Blot } \\
\text { Espectrometría de masas }\end{array}$ & $\begin{array}{l}\text { Se aumenta en el cáncer oral, se relaciona } \\
\text { con la progresión, metástasis y angiogénesis. }\end{array}$ & Dowling, 2008 \\
\hline SAT1 & PCR & Se aumentan en el cáncer oral. & $\begin{array}{l}\text { Brinkmann, } 2012 \\
\text { Elashoff, } 2012\end{array}$ \\
\hline Telomerasa & $\begin{array}{l}\text { PCR } \\
\text { ELISA }\end{array}$ & $\begin{array}{l}\text { Biomarcador de cáncer pero no diferencia } \\
\text { estadio de la lesión. }\end{array}$ & Zhong, 2005 \\
\hline Transferrina & $\begin{array}{l}\text { Eco-2D } \\
\text { MALDI-TOF } \\
\text { Western Blot y ELISA. }\end{array}$ & $\begin{array}{l}\text { El aumento de los niveles salivales de } \\
\text { transferrina se correlaciona con el tamaño } \\
\text { y etapa del tumor. }\end{array}$ & Jou, 2010 \\
\hline
\end{tabular}

ELISA: Ensayo por inmunoabsorción ligado a enzimas; PCR: Reacción en cadena de la polimerasa; SELDITOF: Espectrometría de masas con láser de superficies de una mayor desorción/ionización tiempo de vuelo; Maspin: Inhibidor de la proteasa serina mamaria; S100P: Proteína de unión al calcio; Eco-2D: Electroforesis bidimensional en gel; MALDI-TOF: desorción/ionización láser asistida por matriz. 
Hoffmann (47) afirma que a pesar de que la ET-1 podría mostrar un papel importante en el cáncer oral, sus niveles en la saliva no parecen ser un buen marcador de transformación de neoplasias a un grado maligno.

\section{Ciclina D}

Las ciclinas son una familia de proteínas involucradas en la regulación del ciclo celular. Estas forman complejos con enzimas quinasas dependientes de ciclinas (Cdks) activando en estas últimas su función quinasa. La concentración de las ciclinas varía a lo largo del ciclo celular; cuando su concentración es baja la función de su correspondiente quinasa dependiente de ciclina es inhibida (48).

Durante la fase del ciclo celular G1 temprana la ciclina $\mathrm{D}$ se une a las quinasas dependientes de ciclinas 4 o 6 (Cdk4 o Cdk6) y el complejo resultante libera el freno que impedía la progresión hacia la G1 tardía y, por lo tanto, el paso a la fase S. El complejo ciclina D-Cdk4/6 desarma un potente inhibidor de la progresión del ciclo celular: el formado por la proteína pRB y los factores de transcripción inactivos (49).

Dada la participación de la ciclina $\mathrm{D}$ en el proceso de división celular, se ha sugerido que esta proteína podría participar en el desarrollo de distintos tipos de cáncer. La amplificación y sobreexpresión de la ciclina D1 es importante en el desarrollo de diversos tipos de cáncer, en el oral se correlaciona con la progresión celular de la proliferación tumoral, la metástasis y los pobres pronósticos $(50,51)$.

\section{Ki-67}

Es una proteína nuclear no histónica expresada en las células durante las fases activas del ciclo celular (G1, S, G2, M), y ausente en las células en estado de reposo (G0). Por lo tanto, Ki-67 puede ser empleada para medir crecimiento en los tejidos normales y en las neoplasias malignas. Estudios han demostrado que los altos índices de Ki-67 observados en saliva en pacientes con carcinomas orales de células escamosas estaban correlacionados con la gravedad de la enfermedad y con un mal pronóstico (20).

\section{Galectinas}

Las galectinas constituyen una familia de proteínas extremadamente conservadas a través de la evolución. En función de su propiedad de descifrar glicocódigos específicos, estas proteínas han sido involucradas en un amplio espectro de eventos biológicos. Recientes avances han demostrado que estas proteínas juegan un rol fundamental en procesos relacionados a la regulación de la respuesta inmune, tales como adhesión linfocitaria, crecimiento celular, producción de citoquinas y regulación de la muerte celular programada (52). La intensa inmunoexpresión de galectinas 1,3 , y 7 sugiere la participación de estas proteínas en la carcinogénesis oral y su uso como biomarcadores de comportamiento biológico y progresión del tumor en el carcinoma de células escamosas $(36,53)$.

\section{MARCADORES DE SUPRESIÓN TUMORAL}

\section{p53}

El gen p53 se encuentra en el brazo corto del cromosoma 17 y codifica un factor de transcripción nuclear de 43.7 KDa. Resulta esencial para inducir la respuesta de la célula ante el daño del ADN, deteniendo el ciclo celular en caso de mutación. Éste es un gen supresor tumoral que desempeña un papel importante en apoptosis y control del ciclo celular. Un p53 defectuoso podría permitir que las células proliferen dando por resultado cáncer; alrededor de un $50 \%$ de todos los tumores humanos contienen mutaciones en p53 $(54,55)$.

La inactivación del gen supresor de tumores es importante durante varias etapas de carcinogénesis. La proteína p 53 está frecuentemente mutada en los carcinomas orales de células escamosas $(56,57)$, estas mutaciones pueden servir como biomarcadores muy específicos para la presencia de células tumorales (58). Autores, como Liao (38), indican que la mutación del codón 63 de $p 53$ en la saliva podría ser un biomarcador molecular de los carcinomas orales de células 
escamosas. Además, la cantidad de ADN recuperado de la saliva en la mayoría de los casos es suficientemente grande y su calidad tal que permite la amplificación por PCR (reacción en cadena de la polimerasa), considerando este protocolo como rápido, económico y fácil de realizar, y puede ser útil para estudios epidemiológicos de la carcinogénesis oral.

\section{MARCADORES DE INVASIÓN TUMORAL}

\section{Metaloproteinasas de matriz (MMP)}

Las MMP-2 y MMP-9 son metaloproteinasas que se han demostrado participar en la patogénesis del cáncer, ellas degradan el colágeno tipo IV, el componente principal de la membrana basal, así como otros tipos de colágenos (V, VII y X), elastina y fibronectina; son altamente expresadas en las células del estroma que rodean la frontera de invasión en metástasis de tumores y sus niveles se elevan en el endotelio del tumor y en la orina de pacientes con cáncer (59-61) .Teniendo en cuenta que las MMPs están involucradas en el proceso de metástasis y se asocian a los estadios finales de la lesión, no pueden ser consideradas como un biomarcador de predicción y de diagnóstico precoz del cáncer.

\section{Proteína de unión a calcio S100p}

Las proteínas S100 están localizadas en el citoplasma o el núcleo de una amplia variedad de células y están implicadas en la regulación de diversos procesos celulares tales como la progresión del ciclo celular y la diferenciación celular (62).

El mayor uso de estas proteínas ha sido el de marcadores tumorales. Esto ha permitido resolver diagnósticos de tumores indiferenciados. Lo anterior se ha basado principalmente en la premisa de que estas proteínas participan en diversos procesos celulares claves tanto para la transducción de señales, motilidad y diferenciación celular. Es así como en muchos tumores la expresión de $\mathrm{S} 100$ se ha visto alterada en comparación a los niveles normales, incluso respondiendo a diferentes estadios de la progresión tumoral, relacionándola principalmente con la metástasis tumoral (39).

\section{MARCADORES ENZIMÁTICOS}

\section{La lactato deshidrogenasa (LDH)}

En un medio anaeróbico, el piruvato se reduce a lactato en una reacción reversible catalizada por LDH que utiliza la nicotinamida adenina dinucleótido como coenzima. La LDH es una enzima detectable en el citoplasma de casi todas las células del ser humano, que se convierte extracelular después de la muerte celular. Por lo tanto, su presencia extracelular está siempre relacionada con la necrosis celular y lesión tisular. La similitud entre el perfil de isoformas de la LDH en toda la saliva y el epitelio oral apoya la hipótesis de que la LDH salival es predominantemente de origen extraglandular, por consiguiente, la concentración de LDH en la saliva, como una expresión de necrosis celular, podría ser una indicador específico para las lesiones orales que afectan al integridad de la mucosa oral (63).

La LDH generalmente se encuentra aumentada en varias afecciones orales como la enfermedad periodontal, procesos traumáticos y el cáncer oral (63), en este último ha sido asociada a un pronóstico desfavorable y la recurrencia de las lesiones (64-66). Las isoformas de la LDH más predominante en saliva son la LDH-4 y la LDH-5, del mismo modo autores aseguran que sus nivel son similares en plasma $(67,68)$; teniendo en cuenta esto la LDH es considerado como un biomarcador inespecífico para el cáncer oral debido a que se puede encontrar aumentada por diversos procesos que ocurren en la cavidad oral.

\section{MARCADORES INTRACELULARES}

\section{Cyfra 21-1}

Es un fragmento soluble de la citoqueratina 19 (CK19), la cual es un componente de la proteína del citoesqueleto con un peso molecular de $40 \mathrm{kDa}$, es una citoqueratina de tipo ácido con un $\mathrm{pH}$ isoeléctrico de 5.2. Sheard (69) reportó una elevación de Cyfra 21-1 extracelular concomitantemente con un aumento significativo de Cyfra 21-1 intracelular durante la apoptosis; además, la muerte celular independiente de caspasas se da en presencia de la Z-VAD inhibidor de las caspasas, el cual no permite medir la Cyfra 
21-1. Por lo tanto, la liberación de Cyfra 21-1 se ha sugerido que se produce en las células durante la etapa intermedia de la apoptosis, como consecuencia de la activación de las caspasas y el consecuente aumento extracelular. Según Zhong (28) un aumento en la concentración de Cyfra 21-1 en saliva es un valor clínico potencial para la detección del cáncer oral, así como la predicción de la recurrencia del tumor. Sin embargo, la determinación de la concentración de Cyfra 21-1 en saliva en los pacientes con cáncer oral no es de gran alcance para predicción de la supervivencia.

\section{CONCLUSIONES}

El cáncer oral es una enfermedad asociada a cambios moleculares, genéticos y tisulares; por lo tanto, es indispensable que su diagnóstico se realice precozmente, en este sentido el descubrimiento de biomarcadores en saliva proporciona una herramienta para el diagnóstico, pronóstico y seguimiento al tratamiento.

Existen diferentes biomarcadores salivales reportados, por lo cual se sugiere el análisis de varios biomarcadores en un mismo tiempo para crear un posible perfil bioquímico de cáncer oral, diferencial para cada uno de los estadios de la enfermedad.

\section{BIBLIOGRAFÍA}

1. Balaram P, Sridhar H, Rajkumar T, Vaccarella S, Herrero R, Nandakumar A, et al. Oral cancer in southern India: the influence of smoking, drinking, paan-chewing and oral hygiene. Int $\mathrm{J}$ Cancer 2002;98 (3):440-5.

2. González M. Bases moleculares de la cancerización de cavidad oral. Av Odontoestomatol 2008;24(1):55-60.

3. Bau DT, Tsai MH, Huang CY, Lee CC, Tseng HC, Lo YL, et al. Relationship between polymorphisms of nucleotide excision repair genes and oral cancer risk in Taiwan: evidence for modification of smoking habit. Chin J Physiol 2007;50(6):294300.
4. Brinkmann O, Wong DT. Salivary transcriptome biomarkers in oral squamous cell cancer detection. Adv Clin Chem 2011;55:21-34.

5. Kawakita D, Hosono S, Ito H, Oze I, Watanabe $M$, Hanai $N$, et al. Impact of smoking status on clinical outcome in oral cavity cancer patients. Oral Oncol 2011;48(2):186-91.

6. Guo L, Zhang C, Shi S, Guo X. [Correlation between smoking and the polymorphisms of cytochrome P450 1A1-Msp I and glutathione Stransferase T1 genes and oral cancer]. Hua Xi Kou Qiang Yi Xue Za Zhi 2012;30(2):187-91.

7. Gaitan-Cepeda LA, Peniche-Becerra AG, QuezadaRivera D. Trends in frequency and prevalence of oral cancer and oral squamous cell carcinoma in Mexicans. A 20 years retrospective study. Med Oral Patol Oral Cir Bucal 2011;16(1):e1-5.

8. Chimenos-Kustner E, Font-Costa I, López-López J. Oral cancer risk and molecular markers. Med Oral Patol Oral Cir Bucal 2004;9(5):381-4:77-80.

9. Latini G, De Felice C, Barducci A, Chitano G, Pignatelli A, Grimaldi L, et al. Oral mucosal color changes as a clinical biomarker for cancer detection. Eur J Cancer Prev 2012;21(4):360-66.

10. Bundgaard T, Bentzen SM, Sogaard H. Histological differentiation of oral squamous cell cancer in relation to tobacco smoking. Eur $\mathrm{J}$ Cancer B Oral Oncol 1995;31B(2):118-21.

11. de Jong EP, Xie H, Onsongo G, Stone MD, Chen XB, Kooren JA, et al. Quantitative proteomics reveals myosin and actin as promising saliva biomarkers for distinguishing pre-malignant and malignant oral lesions. PLoS One 2010;5(6): e11148.

12. da Silva SD, Ferlito A, Takes RP, Brakenhoff RH, Valentin MD, Woolgar JA, et al. Advances and applications of oral cancer basic research. Oral Oncol 2011;47(9):783-91.

13. Rosebush MS, Rao SK, Samant S, Gu W, Handorf CR, Pfeffer LM, et al. Oral cancer: enduring 
characteristics and emerging trends. J Tenn Dent Assoc 2011;91(2):24-7; quiz 28-9.

14. Mehrotra R, Gupta DK. Exciting new advances in oral cancer diagnosis: avenues to early detection. Head Neck Oncol 2011;3:33.

15. Humphrey SP, Williamson RT. A review of saliva: normal composition, flow, and function. J Prosthet Dent 2001;85(2):162-9.

16. Huang CM. Comparative proteomic analysis of human whole saliva. Arch Oral Biol 2004;49(12): 951-62.

17. Martins C, Castro GF, Siqueira MF, Xiao Y, Yamaguti PM, Siqueira WL. Effect of dialyzed saliva on human enamel demineralization. Caries $\operatorname{Res} 2013 ; 47(1): 56-62$.

18. Schipper RG, Silletti E, Vingerhoeds MH. Saliva as research material: biochemical, physicochemical and practical aspects. Arch Oral Biol 2007;52 (12):1114-35.

19. Hofman LF. Human saliva as a diagnostic specimen. J Nutr 2001;131(5):1621S-5S.

20. Shpitzer T, Hamzany Y, Bahar G, Feinmesser R, Savulescu D, Borovoi I, et al. Salivary analysis of oral cancer biomarkers. Br J Cancer 2009;101 (7):1194-8.

21. Szanto I, Mark L, Bona A, Maasz G, Sandor B, Gelencser G, et al. High-throughput screening of saliva for early detection of oral cancer: a pilot study. Technol Cancer Res Treat 2012;11(2):181-8.

22. Chen YC, Li TY, Tsai MF. Analysis of the saliva from patients with oral cancer by matrix-assisted laser desorption/ionization time-of-flight mass spectrometry. Rapid Commun Mass Spectrom 2002;16(5):364-9.

23. Nagler RM. Saliva as a tool for oral cancer diagnosis and prognosis. Oral Oncol 2009;45 (12):1006-10.

24. Chiappin S, Antonelli G, Gatti R, De Palo EF. Saliva specimen: a new laboratory tool for diagnostic and basic investigation. Clin Chim Acta 2007;383(1-2):30-40.
25. Al Kawas S, Rahim ZH, Ferguson DB. Potential uses of human salivary protein and peptide analysis in the diagnosis of disease. Arch Oral Biol 2012;57(1):1-9.

26. Hu S, Yu T, Xie Y, Yang Y, Li Y, Zhou X, et al. Discovery of oral fluid biomarkers for human oral cancer by mass spectrometry. Cancer Genomics Proteomics 2007;4(2):55-64.

27. Nagler R, Bahar G, Shpitzer T, Feinmesser R. Concomitant analysisof salivary tumor markers a new diagnostic tool for oral cancer. Clin Cancer Res 2006;12:3979-84.

28. Zhong LP, Zhang CP, Zheng JW, Li J, Chen WT, Zhang ZY. Increased Cyfra 21-1 concentration in saliva from primary oral squamous cell carcinoma patients. Arch Oral Biol 2007;52(11):107987.

29. Liu J, Duan Y. Saliva: A potential media for disease diagnostics and monitoring. Oral Oncol 2012;48(7):569-77.

30. Bernardes V, Gleber-Netto F, Sousa S, Silva T, Aguiar M. Clinical significance of EGFR, Her-2 and EGF in oral squamous cell carcinoma: a case control study. J Exp Clin Cancer Res 2010;29:29-40.

31. Elashoff D, Zhou H, Reiss J, Wang J, Xiao H, Henson B, et al. Prevalidation of salivary biomarkers for oral cancer detection. Cancer Epidemiol Biomarkers Prev 2012;21(4):664-72.

32. Xiao H, Zhang L, Zhou H, Lee J, Garon E, Wong D. Proteomic analysis of human saliva from lung cancer patients using two-dimensional difference gel electrophoresis and mass spectrometry. Mol Cell Proteomics 2012;11 (2):M111.012112.

33. Metzger K, Angres G, Maier H, Lehmann WD. Lipoxygenase products in human saliva: patients with oral cancer compared to controls. Free Radic Biol Med 1995;18(2):185-94.

34. Shintani S, Hamakawa H, Ueyama Y, Hatori M, Toyoshima T. Identification of a truncated cystatin SA-I as a saliva biomarker for oral squamous cell 
carcinoma using the SELDI ProteinChip platform. Int J Oral Maxillofac Surg 2010;39(1):68-74.

35. Cheng YS, Rees T, Jordan L, Oxford L, O'Brien $\mathrm{J}$, Chen HS, et al. Salivary endothelin-1 potential for detecting oral cancer in patients with oral lichen planus or oral cancer in remission. Oral Oncol 2011;47(12):1122-6.

36. Alves PM, Godoy GP, Gomes DQ, Medeiros AM, de Souza LB, da Silveira EJ, et al. Significance of galectins-1, $-3,-4$ and -7 in the progression of squamous cell carcinoma of the tongue. Pathol Res Pract 2011;207(4):236-40.

37. Shpitzer T, Bahar G, Feinmesser R, Nagler RM. A comprehensive salivary analysis for oral cancer diagnosis. J Cancer Res Clin Oncol 2007;133(9): 613-7.

38. Liao PH, Chang YC, Huang MF, Tai KW, Chou MY. Mutation of p53 gene codon 63 in saliva as a molecular marker for oral squamous cell carcinomas. Oral Oncol 2000;36(3):272-6.

39. Dowling P, Wormald R, Meleady P, Henry M, Curran A, Clynes M. Analysis of the saliva proteome from patients with head and neck squamous cell carcinoma reveals differences in abundance levels of proteins associated with tumour progression and metastasis. J Proteomics 2008;71(2):168-75.

40. Zhong LP, Chen GF, Xu ZF, Zhang X, Ping FY, Zhao SF. Detection of telomerase activity in saliva from oral squamous cell carcinoma patients. Int J Oral Maxillofac Surg 2005;34(5):566-70.

41. Jou YJ, Lin CD, Lai CH, Chen CH, Kao JY, Chen SY, et al. Proteomic identification of salivary transferrin as a biomarker for early detection of oral cancer. Anal Chim Acta 2010;681(1-2): 41-8.

42. Brinkmann O, Kastratovic DA, Dimitrijevic MV, Konstantinovic VS, Jelovac DB, Antic J, et al. Oral squamous cell carcinoma detection by salivary biomarkers in a Serbian population. Oral Oncol 2012;47(1):51-5.
43. Allsopp RC, Chang E, Kashefi-Aazam M, Rogaev EI, Piatyszek MA, Shay JW, et al. Telomere shortening is associated with cell division in vitro and in vivo. Exp Cell Res 1995;220(1):194-200.

44. Ries JC, Hassfurther E, Steininger H, Kloss FR, Wiltfang J, Girod SC, et al. Correlation of telomerase activity, clinical prognosis and therapy in oral carcinogenesis. Anticancer Res 2001;21 (2A): 1057-63.

45. Xiang S, Denver R, Bailey M, Krum H. Physiologic determinants of endothelin concentrations in human saliva. Clin Chem 2003;49(12):2012-9.

46. Pickering V, Jordan RC, Schmidt BL. Elevated salivary endothelin levels in oral cancer patients a pilot study. Oral Oncol 2007;43(1):37-41.

47. Hoffmann RR, Yurgel LS, Campos MM. Endothelins and their receptors as biological markers for oral cancer. Oral Oncol 2010;46(9): 644-7.

48. Tajara EH. Oral cancer and cyclins. Int J Oral Maxillofac Surg 2004;33(5):518; author reply 19.

49. Miyashita H, Uchida T, Mori S, Echigo S, Motegi K. Expression status of Pin1 and cyclins in oral squamous cell carcinoma: Pin1 correlates with Cyclin D1 mRNA expression and clinical significance of cyclins. Oncol Rep 2003;10(4): 1045-8.

50. Adjei AA. Targeting multiple signal transduction pathways in lung cancer. Clin Lung Cancer 2005; 7 Suppl 1:S39-44.

51. Wang L, Liu T, Nishioka M, Aguirre RL, Win SS, Okada N. Activation of ERK1/2 and cyclin D1 expression in oral tongue squamous cell carcinomas: relationship between clinicopathological appearances and cell proliferation. Oral Oncol 2006;42(6):625-31.

52. Kasamatsu A, Uzawa K, Nakashima D, Koike H, Shiiba $M$, Bukawa $H$, et al. Galectin-9 as a regulator of cellular adhesion in human oral squamous cell carcinoma cell lines. Int $\mathrm{J}$ Mol Med 2005;16 (2):269-73. 
53. Wei KJ, Pan HY, Yang X, Zhong LP, Ye DX, Zhang ZY. [Expression of galectin-1 in carcinogenesis of oral mucosal epithelium]. Zhonghua Kou Qiang Yi Xue Za Zhi 2011;46(9):524-7.

54. Jing G, Lv K, Jiao X. The p53 Codon 72 Polymorphism and the Risk of Oral Cancer in a Chinese Han Population. Genet Test Mol Biomarkers 2012.

55. Malecki K, Glinski B, Mucha-Malecka A, Roszkowski K, Hetnal M. [Predictive and prognostic value of p53, Ki-67 and EGFR in patients with advanced oral cavity and oropharyngeal cancer treated with induction chemotherapy]. Przegl Lek 2012;69(1):5-8.

56. Mroz EA, Rocco JW. Functional p53 status as a biomarker for chemotherapy response in oralcavity cancer. J Clin Oncol 2010;28(5):715-7.

57. Khan Z, Tiwari RP, Mulherkar R, Sah NK, Prasad GB, Shrivastava BR, et al. Detection of survivin and p53 in human oral cancer: correlation with clinicopathologic findings. Head Neck 2009;31 (8):1039-48.

58. Liu M, Lawson G, Delos M, Jamart J, Ide C, Coche $\mathrm{E}$, et al. Predictive value of the fraction of cancer cells immunolabeled for proliferating cell nuclear antigen or Ki67 in biopsies of head and neck carcinomas to identify lymph node metastasis: comparison with clinical and radiologic examinations. Head Neck 2003;25(4):280-8.

59. Pories SE, Zurakowski D, Roy R, Lamb CC, Raza S, Exarhopoulos A, et al. Urinary metalloproteinases: noninvasive biomarkers for breast cancer risk assessment. Cancer Epidemiol Biomarkers Prev 2008; 17(5):1034-42.

60. Smith ER, Zurakowski D, Saad A, Scott RM, Moses MA. Urinary biomarkers predict brain tumor presence and response to therapy. Clin Cancer Res 2008;14(8):2378-86.

61. Chen L, Sun B, Zhang S, Zhao X, He Y, Zhao S, et al. Influence of microenvironments on microcirculation patterns and tumor invasion- related protein expression in melanoma. Oncol Rep 2009;21(4):917-23.

62. Arumugam T, Ramachandran V, Gomez SB, Schmidt AM, Logsdon CD. S100P-Derived RAGE Antagonistic Peptide Reduces Tumor Growth and Metastasis. Clin Cancer Res 2012;18(16):4356-64.

63. De La Pena VA, Diz Dios P, Tojo Sierra R. Relationship between lactate dehydrogenase activity in saliva and oral health status. Arch Oral Biol 2007;52(10):911-5.

64. Schneider J. Tumor markers in detection of lung cancer. Adv Clin Chem 2006;42:1-41.

65. Duffy MJ, Crown J. A personalized approach to cancer treatment: how biomarkers can help. Clin Chem 2008;54(11):1770-9.

66. Culine S. Prognostic factors in unknown primary cancer. Semin Oncol 2009;36(1):60-4.

67. Alonso de la Pena V, Diz Dios P, Lojo Rocamonde S, Tojo Sierra R, Rodriguez-Segade S. A standardised protocol for the quantification of lactate dehydrogenase activity in saliva. Arch Oral Biol 2004;49(1):23-7.

68. Nagler RM, Lischinsky S, Diamond E, Klein I, Reznick AZ. New insights into salivary lactate dehydrogenase of human subjects. J Lab Clin Med 2001;137(5):363-9.

69. Sheard MA, Vojtesek B, Simickova M, Valik D. Release of cytokeratin-18 and -19 fragments (TPS and CYFRA 21-1) into the extracellular space during apoptosis. J Cell Biochem 2002;85(4):670-7.

\section{CORRESPONDENCIA}

Meisser Vidal Madera Anaya

Universidad de Cartagena, Campus de la Salud

Facultad de Odontología, Zaragocilla

Cartagena de Indias

Bolívar. Colombia

Correo electrónico: meissermadera@gmail.com 